See Article page 322

\section{Commentary: Preparation is half the battle - preclosure devices for peripheral venoarterial extracorporeal oxygenation}

\author{
Charles Laurin, MD, and Dimitri Kalavrouziotis, MD
}

Percutaneous venoarterial extracorporeal membrane oxygenation (VA-ECMO) can be life-saving in profound cardiogenic shock and ongoing cardiopulmonary resuscitation (ie, extracorporeal cardiopulmonary resuscitation). Percutaneous autosuture devices have gained popularity in recent years and are now widely used for transfemoral transcatheter aortic valve replacement (TAVR) and aortic endografting. Whether such devices may impact the outcomes of VA-ECMO via the femoral artery remains unknown.

In this issue of the Journal, Chandel and colleagues ${ }^{1}$ report their experience with the Perclose ProGlide device (Abbott, Chicago, Ill) in VA ECMO via the femoral artery. The autosutures were placed as a "preclosure" strategy, that is, before arterial cannula placement. The preclosure group is a large cohort of 50 patients that was compared to patients who were decannulated via surgical cutdown. Five failures occurred in the preclosure group. However, the preclosure strategy was associated with a significant reduction in groin infection rates, bleeding, and limb complications, which can be devastating following peripheral VA-ECMO. ${ }^{2,3}$

Chandel and colleaugues ${ }^{1}$ demonstrate the safety and feasibility of the preclosure strategy in femorally cannulated patients on VA-ECMO. This is great news for surgeons, as few enjoy returning to the operating room, cutting down on percutaneously cannulated femoral

\footnotetext{
From the Department of Cardiac Surgery, Quebec Heart and Lung Institute, Quebec City, Quebec, Canada.

Disclosures: The authors reported no conflicts of interest.

The Journal policy requires editors and reviewers to disclose conflicts of interest and to decline handling or reviewing manuscripts for which they may have a conflict of interest. The editors and reviewers of this article have no conflicts of interest.

Received for publication Sept 8, 2021; revisions received Sept 8, 2021; accepted for publication Sept 10, 2021; available ahead of print Sept 16, 2021.

Address for reprints: Dimitri Kalavrouziotis, MD, Department of Cardiac Surgery, Quebec Heart and Lung Institute, 2725 Chemin Sainte-Foy, Quebec City, Quebec, Canada G1V 4G5 (E-mail: dimitri.kalavrouziotis@criucpq.ulaval.ca).

JTCVS Techniques 2021;10:333-4

2666-2507

Copyright (C) 2021 The Author(s). Published by Elsevier Inc. on behalf of The American Association for Thoracic Surgery. This is an open access article under the CC BY-NC-ND license (http://creativecommons.org/licenses/by-nc-nd/4.0/).

https://doi.org/10.1016/j.xjtc.2021.09.020
}

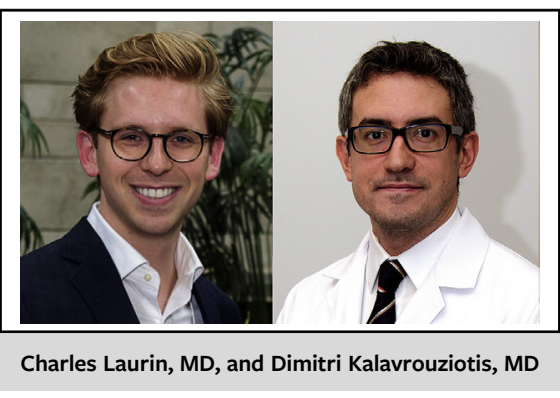

CENTRAL MESSAGE

Preclosure devices at the time of peripheral ECMO deployment may decrease bleeding and limb complications and avoid surgical cut-down at decannulation.

vessels, decannulating, and often having to perform various degrees of vascular repair with or without proximal and distal vessel control. However, the main problem of the comparative analysis is that, in fact, the 2 groups (preclosure vs surgical cutdown) are not comparable. This is because the surgical cutdown group may be a sicker cohort who would be ineligible for preclosure, thus explaining the better outcomes in the latter. Not surprisingly, among the 18 patients with extracorporeal cardiopulmonary resuscitation, which is a well-described negative prognostic factor, 14 $(78 \%)$ were managed by direct cannulation without preclosure.

Transfemoral TAVR has taught us that common femoral artery (CFA) calcification is an important determinant of success of preclosure devices. Surface ultrasound or fluoroscopy are luxuries that are seldom available during ECMO deployment but should be encouraged in "semi-elective" situations to identify the CFA and make sure the puncture isn't below the bifurcation, and to avoid areas of calcification. It remains unclear to what extent imaging was used in the study by Chandel and colleagues, ${ }^{1}$ and whether their results are reproducible when using larger arterial cannulas, as the average cannula size was $17 \mathrm{Fr}$.

Another unanswered issue relates to the fact that late vascular complications may be greater after autosuture devices compared with surgical cutdown." The "Perclose sign" (residual stenosis after tying down the autosutures postsheath removal) is not uncommon after TAVR, and certainly depends on how hard one looks for it. The 
significance of this in the ECMO population, which is, on average, 20 to 30 years younger than the patients who undergo TAVR, remains unclear.

Our last point relates to the nearly $25 \%$ bleeding rate in the surgical cutdown group. Exposure with proximal and distal control of the CFA under direct surgical visualization should preclude massive bleeding. The authors merged all Bleeding Academic Research Consortium (BARC) 2 to 4 bleeding together and therefore did not exclude the minor bleeding cases from the end point.

In summary, the study by Chandel and colleagues ${ }^{1}$ provides an interesting proof-of-concept that suggests that a preclosure strategy can be safe and effective in femoral VA-ECMO. Late follow-up data are needed to determine whether these results are sustained over time.

\section{References}

1. Chandel A, Desai M, Ryan LP, Clevenger L, Speir AM, Singh R. Preclosure technique versus arterial cutdown after percutaneous cannulation for venorterial extracorporeal membrane oxygenation. J Thorac Cardiovasc Surg Tech. 2021;10: 322-30.

2. Jia D, Yang IX, Ling RR, Syn N, Poon WH, Murughan K, et al. Vascular complications of extracorporeal membrane oxygenation: a systematic review and metaregression analysis. Crit Care Med. 2020;48:e1269-77.

3. Lamb KM, DiMuzio PJ, Johnson A, Batista P, Moudgill N, McCullough M, et al Arterial protocol including prophylactic distal perfusion catheter decreases limb ischemia complications in patients undergoing extracorporeal membrane oxygenation. J Vasc Surg. 2017;65:1074-9.

4. Vierhout BP, Pol RA, El Moumni M, Zeebregts CJ. Editor's choice-arteriotomy closure devices in EVAR, TEVAR, and TAVR: a systematic review and metaanalysis of randomised clinical trials and cohort studies. Eur J Vasc Endovasc Surg. 2017;54:104-15.

5. Nakamura M, Chakravarty T, Jilaihawi H, Doctor N, Dohad S, Fontana G, et al. Complete percutaneous approach for arterial access in transfemoral transcatheter aortic valve replacement: a comparison with surgical cut-down and closure. Catheter Cardiovasc Interv. 2014;84:293-300. 